\title{
The High Vacuum Cleaving Passivation Characteristic on 980nm Diode Laser
}

\author{
Tiansheng ZHAO, Zaijin LI*, Te LI, Peng LU, Yi QU, Baoxue BO, Guojun LIU, \\ Xiaohui MA, Yong WANG
}

National Key Lab on High Power Semiconductor Lasers, Changchun University of Science and Technology, Changchun 130022, China

*Corresponding author:lizaijin@126.com

Keywords: Facet; Passivation; Diode laser

\begin{abstract}
The high vacuum cleaving passivation characteristic on 980nm diode laser is presented. In this research, diode lasers are cleaved in high vacuum cleaving system, and then coated with thin ZnSe passivation layer in the front and the back facet. The function of the passivation layer is to protect diode lasers facet, and prevent impurity particles diffusing into the facet. Finally optical coatings are applied to the AR and HR facets of 5\% and 98\% respectively in the front and the back facets. The test results of diode lasers output power show that the output power with ZnSe passivation layer method is $9 \%$ higher than Si passivation layer, and 21\% higher than that uncoated with passivation layer. The diode of uncoated passivation layer is failed when input current is 3.9A, and the diode coated with Si passivation layer is failed when input current is 4.6A, the final failed of the diode is coated ZnSe passivation layer. In conclusion, the method of coated ZnSe passivation layer in high vacuum cleaving system on the diode lasers facet can effectively prevent the catastrophic optical mirror damage, and increase the output power of diode lasers.
\end{abstract}

\section{Introduction}

Nowadays, Diode lasers are widely used in many fields, pumping fibre lasers, medical treatments, processing materials and so on[1]. Diode Lasers as the pumping sources of fibre lasers are affected by output light power, input current and thermal effect during their operation [2-3]. diode lasers facets is easy to generate light absorption, and the light absorption results to generate heat, the heat will increase laser diodes degradation, and even lead to catastrophic optical mirror damage [4], which makes the diode lasers failed. In order to improve the output power of diode lasers, many facets coating technology are developed. One is facet sulfur disposing technology. The diode lasers facets react with sulfur compounds, remove the natural facets oxide layer and generate a stable sulfide layer to protect facets. Additionally, the current non injection zone at front facet is introduced to limit the current flow into facets, and reduce facets carriers' concentration [5]. Furthermore, the non absorption window technology is introduced to improve diode lasers characteristics[6]. In these facets technologies, facet sulfur passivation technology is not stable enough[7]. Non absorption window technology involves the second epitaxial growth technology, which is difficult in technology and has a low reproducibility[8]. The high vacuum facet passivation technology is simple and has a good consistency. The output power of a single emitter of 980nm diode laser is up to $4.3 \mathrm{~W}$ by using the high vacuum cleaving facet passviation technology.

\section{High vacuum cleaving system}

The high vacuum cleaving system contain four Chambers: Loadlock Chamber, Buffer Chamber, Cleaving Chamber and Deposition Chamber. The vacuum level of Loadlock Chamber is $2 \times 10^{-8}$ torr, the vacuum level of Buffer Chamber is $5 \times 10^{-10}$ torr, the vacuum level of Cleaving Chamber is $5 \times 10^{-10}$ torr, and the vacuum level of Deposition Chamber is $2 \times 10^{-10}$ torr. A mechanical fixture was developed that is capable of cleaving diode laser bars in Cleaving Chamber. The bars can have widths ranging from $1000 \mu \mathrm{m}$ to $4000 \mu \mathrm{m}$ and thicknesses ranging from 100 to $400 \mu \mathrm{m}$. The diode 
lasers were not exposed to air until the facet passivation layer was completed, and this method can effectively prevent the catastrophic optical damage, and increase the output power of diode lasers.

\section{The design of epitaxial wafer structure}

The epitaxial material of $980 \mathrm{~nm}$ semiconductor laser is based on InGaAs/AlGaAs. The structure is shown in Figure 1. Its epitaxial material structure uses the gradient index waveguide. Epitaxial material structure includes: $2 \mu \mathrm{m} \mathrm{Al}_{0.3} \mathrm{Ga}_{0.7} \mathrm{As}$ (Si doping concentration is $1 \times 10^{18} \mathrm{~cm}^{-3}$ ) cover layer grown on the $\mathrm{n}^{+}-\mathrm{GaAs}$ (Si doping concentration is $2 \times 10^{18} \mathrm{~cm}^{-3}$ ) substrate; And then $0.11 \mu \mathrm{m}$ undoped $\mathrm{Al}_{\mathrm{x}} \mathrm{Ga}_{1-\mathrm{x}} \mathrm{As}$ waveguide structure, in which $\mathrm{Al}$ composition changes linearly from 0.1 to 0.3 ; $8 \mathrm{~nm} \mathrm{In}_{0.2} \mathrm{Ga}_{0.8} \mathrm{As}$ active region; $0.11 \mu \mathrm{m}$ undoped $\mathrm{Al}_{\mathrm{x}} \mathrm{Ga}_{1-\mathrm{x}}$ As waveguide structure, in which $\mathrm{Al}$ composition changes linearly from 0.3 to $0.1 ; 2 \mu \mathrm{m} \mathrm{Al}_{0.3} \mathrm{Ga}_{0.7} \mathrm{As}$ ( $\mathrm{Zn}$ doping concentration is $2 \times 10^{18} \mathrm{~cm}^{-3}$ ) cover layer; $0.1 \mu \mathrm{m}$ highly doped $\mathrm{p}^{+}-\mathrm{GaAs}\left(\mathrm{Zn}\right.$ doping concentration is $2 \times 10^{19} \mathrm{~cm}-3$ ) contact layer.

\begin{tabular}{|c|}
\hline $\mathbf{p}^{+}$-GaAs contact layer \\
\hline p- $\mathbf{A l}_{0.3} \mathbf{G a}_{0.7} \mathbf{A s}$ cladding layer \\
\hline $\mathrm{p}-\mathrm{Al}_{\mathrm{x}} \mathrm{Ga}_{1-\mathrm{x}} \mathrm{As}$ waveguide layer \\
\hline $\mathrm{In}_{0.2} \mathbf{G a}_{0.8}$ As SQW \\
\hline $\mathrm{n}$-Al $\mathrm{G}_{\mathrm{G}} \mathrm{a}_{1-\mathrm{A}} \mathrm{As}$ waveguide layer \\
\hline $\mathbf{n}-\mathbf{A l}_{0.3} \mathbf{G a}_{0.7}$ As cladding layer \\
\hline $\mathbf{n}^{+}$-GaAs substrate \\
\hline
\end{tabular}

Fig.1 epitaxial material structure of $980 \mathrm{~nm}$ semiconductor laser diode

\section{The fabrication of the facet passivation layer}

The laser wafer is cleaved into the bars with cavity length of $1000 \mu \mathrm{m}$ in the high vacuum cleaving system and coated passivation layer. In order to compare the influence of the laser diodes output light power with different facet coating methods and the feasibility of the new coating method used, the bars are divided into three groups. The first group of the bars is uncoated passivation layer on the facet. The second group of bars is coated with 10nm Si passivation layer. The third group of bars is coated with $10 \mathrm{~nm}$ ZnSe passivation layer. Finally all laser bars are put into the electron beam vacuum coating machine coated with AR and HR facets of 5\% and 98\% respectively in front and back facet. Subsequently, the laser bars are cleaved into single emitters, and the single emitter was sealed onto the oxygen-free copper heat sink with In-Sn mixture solder. Finally, the output light power was tested with laser diodes parameter measure instrument.

\section{Results and discussions}

In fig.2, output power versus input current of laser diode at uncoated passivation layer, Si passivation layer and ZnSe passivation layer are shown. It can be seen from the measure curve that the diodes with uncoated passivation layer are failed at first, followed by the diode with $\mathrm{Si}$ passivation layer, and the diodes with ZnSe passivation layer are failed at last. In conclusion, the 
ZnSe passivation layer coated on the laser facet can effectively prevent damage. The measure results of diode lasers output power show that output power of the ZnSe passivation layer is 9\% higher than Si passivation layer, and 21\% higher than uncoated passivation layer. The ZnSe passivation layer coated on the diode laser facet can increase the output power.

The diode of uncoated passivation layer is failed when current is 3.9A. Light is absorbed on the facets, the heat is generated and the bond of the molecules is breaking. The Ga-O and As-O combinations are formed, oxidation is generated on the facets. With the absorption increasing, the temperature is continuously rised, when the temperature reaches the melting point of the wafer material, result to damage. Finally the diode is failed. The diode with coated Si passivation layer is failed when current is 4.6A. It means that the diode of coated Si passivation layer is more effective to prevent damage than that of uncoated passivation layer. The diode with ZnSe passivation layer is failed when current is 4.8A, which means the coated ZnSe passivation layer is the most effective in the three groups. The diode with Si passivation layer coated is failed before the diode with $\mathrm{ZnSe}$ passivation layer. The band gap of $\mathrm{Si}$ is $1.12 \mathrm{eV}$, and the band gap of $\mathrm{GaAs}$ is $1.42 \mathrm{eV}$. Because of the band gap of Si is smaller than, so it is easy to form a trap at the facets, electrons and holes can be recombine, and then generate absorption, finally the diode is failed. The band gap of ZnSe material is $2.75 \mathrm{eV}$, that is bigger than GaAs. It acts as an electrical barrier for electrons and holes to reach the facets, and eliminates the facet recombination absorption, and improves the COMD threshold of laser diode.

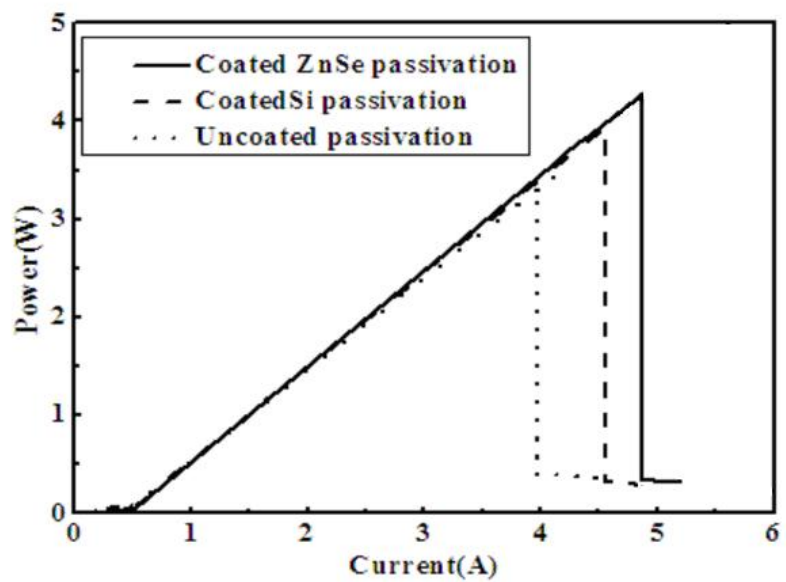

Fig.2 Output light power versus drive current of laser diode at uncoated passivation, Si passivation and ZnSe passivation

\section{Conclusion}

The laser diode coated with ZnSe passivation layer has the highest COMD threshold. The test results show that output light power of the ZnSe passivation is 9\% higher than Si passivation and $21 \%$ higher than uncoated passivation, which means that the method of high vacuum cleaving facet coating ZnSe passivation layer is effective to raise the output light power of high power laser diodes.

\section{Acknowledgement}

In this paper, the research was sponsored by NSAF (NO. U1330136), the National Natural Science Foundation of China (NO. 61107054), National Key Laboratory Foundation (233360) and the Jilin Province Science and Technology Development Plan Item (NO. 201201124).

\section{References}

[1] Britta Leonhäuser; Heiko Kissel ; Andreas Unger; Bernd Köhler; Jens Biesenbach Proc. SPIE 8965, 896506, 2014. 
[2] Lijun Wang ; Cunzhu Tong ; Hangyu Peng ; Jun Zhang Proc. SPIE 8796, 87961N, 2012.

[3] Yuri Berk; Yoram Karni ; Genady Klumel ; Yaakov Openhaim ; Shalom Cohen ; Dan Yanson,Proc. SPIE. 7918, 79180W, 2011.

[4] Uwe Brauch, Peter Loosen, and Hans Opower,Springer-Series, Topics in Applied Physics. 78, 303, 2000.

[5]R. Hülsewede, H. Schulze, J. Sebastian, D. Schröder, J. Meusel: SPIE, vol. 6456, 645607, 2007.

[6]R. V. Ghita, M. F. Lazarescu, A. S. Manea, C. Logofatu, E.Vasile: SPIE, vol. 5581, 268,2004.

[7]F. Rinner, J. Rogg, M. T. Kelemen, M. Mikulla, G. Weimann:J. Appl. Phys. 93(3), 1848,2003.

[8]H. Horie, Y. Yamamoto, N. Arai, H. Ohta: IEEE photonics technology letters, vol. 12(1), 13, 2000. 